\title{
Relay Station Access Link Spectral Efficiency Optimization through SO of Macro BS Tilts
}

\author{
Ali Imran, Muhammed Ali Imran and Rahim Tafazolli; Centre for Communication and Systems Research \\ University of Surrey, Guildford, United Kingdom. GU2 7XH. \\ email: \{A.Imran, M.Imran, R.Tafazolli\}@ surrey.ac.uk
}

\begin{abstract}
In this paper we present a novel framework for spectral efficiency enhancement on the access link between relay stations and their donor base station through Self Organization (SO) of system-wide BS antenna tilts. Underlying idea of framework is inspired by SO in biological systems. Proposed solution can improve the spectral efficiency by upto $1 \mathrm{bps} / \mathrm{Hz}$.
\end{abstract}

\section{INTRODUCTION}

Future Wireless Cellular Systems (WCS) are marked by increasing use of Relay Stations (RS) to meet the ever rising demand for better QoS and capacity. Compared to conventional BS's (macro or micro cells), RS (usually Pico or Femto cells) are generally cheaper solution to extend coverage and capacity of WCS [1]. RS's cost effectiveness lies in the fact that they are constituted of very basic hardware needed to only relay the user traffic to their donor BS. Therefore, most of the complexity and cost remains confined to BS's. However, RS need additional radio resources to relay the traffic to their donor BS through an over the air access link. Such additional partitioning of resources is bound to have negative impact on the over all spectrum reuse efficiency of the system and hence capacity. Therefore, it is very desirable to optimise the spectrum efficiency of access link so that less fraction of radio resources have to be allocated to the access link and more radio resources are available to provide services to the users. In this paper we present a novel framework for spectral efficiency enhancement on the access link through optimization of system-wide BS antenna tilts in a distributed manner.

A significant number of works have embarked on tilt optimization for coverage and capacity enhancement in macro cellular networks [2]-[4]. However to the best of our knowledge, this concept of spectral efficiency enhancement on access link through BS antenna tilt adaptation is novel and the solution presented here is first attempt in this particular direction. Furthermore, another novelty of solution is its SO nature enabled by its distributed design inspired from SO in biological systems; which makes it very suitable solution for emerging WCS e.g. LTE-A. The rest of paper is organised as follows. In section II we present system model, assumptions and problem formulation. In order to achieve a SO solution, in section III we propose a way to decompose the system-wide problem into local subproblems as inspired by SO systems in nature. Solution methodology for local subproblems is also presented in this section. Section IV concludes this paper by presenting numerical results to demonstrate the potential of

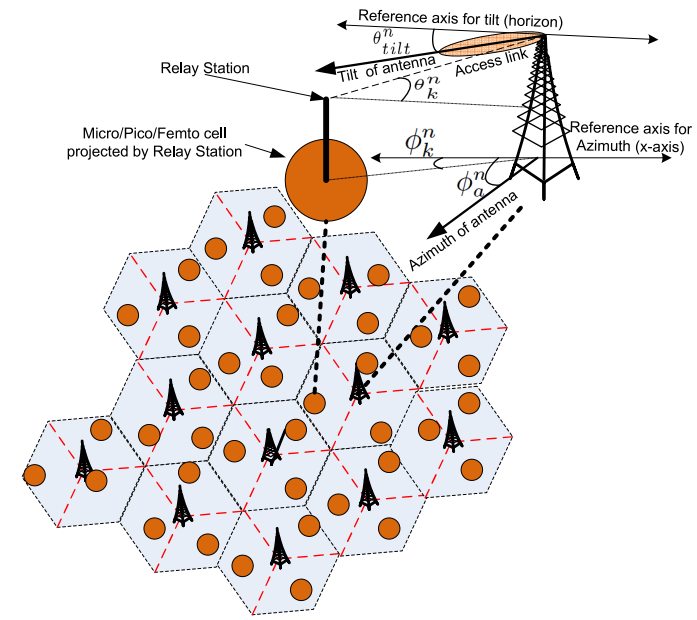

Fig. 1. System model for problem formulation. Small circles show location of Relay Stations based cells within each sector (macro cell).

the proposed solution. Pragmatic implementation aspects in context of LTE and LTE-A are also highlighted in this section.

\section{System Model and Problem Formulation}

We consider a multicellular WCS with each BS having three sectors as shown in figure 1 . Let $\mathcal{N}$ denote the set of locations of the transmission antenna of all sectors and $\mathcal{K}$ denote the set of points representing arbitrary locations in the system e.g. location of RSs. The geometric Signal to Interference Ratio i.e. SIR -i.e.SIR with only pathloss based channel model with no fading and correlation assumed-perceived at a location $k$ being served by $n^{\text {th }}$ sector can be given as:

$$
\gamma_{k}^{n}=\frac{P^{n} G_{k}^{n} \alpha\left(d_{k}^{n}\right)^{-\beta}}{\sum_{\forall m \in \mathcal{N} \backslash n}\left(P^{m} G_{k}^{m} \alpha\left(d_{k}^{m}\right)^{-\beta}\right)} m, n \in \mathcal{N}, k \in \mathcal{K}
$$

where $P$ is transmission power, $d$ is distance $\alpha$ and $\beta$ are pathloss coefficient and exponent. $G$ is antenna gain and for 3GPP WCS like LTE and LTE-A it is modelled in $\mathrm{dB}$ as:

$$
\begin{aligned}
G_{k}^{n}= & \lambda_{v}\left(G_{\max }-\min \left(12\left(\frac{\theta_{k}^{n}-\theta_{\text {tilt }}^{n}}{B_{v}}\right)^{2}, A_{\max }\right)\right)+ \\
& \lambda_{h}\left(G_{\max }-\min \left(12\left(\frac{\phi_{k}^{n}-\phi_{a}^{n}}{B_{h}}\right)^{2}, A_{\max }\right)\right)
\end{aligned}
$$

where $\theta_{\text {tilt }}^{n}$ and $\phi_{a}^{n}$ are tilt and azimuth angles of $n^{\text {th }}$ sector with respect to the horizon and $x$-axis used as reference. 
Similarly, $\theta_{k}^{n}$ and $\phi_{k}^{n}$ are angles of $k^{t h}$ location in $n^{\text {th }}$ sector, with respect to horizon and positive $\mathrm{x}$-axis respectively, as illustrated in figure 1. Subscripts $h, a$ and $v$ denote horizontal, azimuth and vertical, respectively. $B$ represents beamwidth and $\lambda$ is weighting factor to weight horizontal and vertical beam pattern of the antenna in 3D antenna model. For sake of simplicity we can neglect the the maximum attenuation factor $A_{\max }$ in (2). Without loss of generality we assume maximum gain of $0 \mathrm{~dB}$. Thus by putting $G_{\max }=0 \mathrm{~dB}$ in (2), converting it from $d B$ to linear it can be simplified as:

$$
G_{k}^{n}=10^{-1.2\left(\lambda_{v}\left(\frac{\theta_{k}^{n}-\theta_{t i l t}^{n}}{B_{v}}\right)^{2}+\lambda_{h}\left(\frac{\phi_{k}^{n}-\phi_{a}^{n}}{B_{h}}\right)^{2}\right)}
$$

For ease of expression we use following substitutions:

$$
c_{k}^{l}=\frac{B_{v}^{2} \lambda_{h}}{\lambda_{v}}\left(\frac{\phi_{k}^{l}-\phi_{a}^{l}}{B_{h}}\right)^{2} ; h_{k}^{m}=\alpha\left(d_{k}^{m}\right)^{-\beta} ; \mu=\frac{-1.2 \lambda_{v}}{B_{v}^{2}}
$$

Assuming that all the BS have same transmit power and using (3) and (4) in (1), the SIR can be finally written as:

$$
\gamma_{k}^{n}=\frac{h_{k}^{n} 10^{\mu\left(\left(\theta_{k}^{n}-\theta_{t i l t}^{n}\right)^{2}+c_{k}^{n}\right)}}{\sum_{\forall m \in \mathcal{N} \backslash n}\left(h_{k}^{m} 10^{\mu\left(\left(\theta_{k}^{m}-\theta_{t i l t}^{m}\right)^{2}+c_{k}^{m}\right)}\right)}
$$

Note that $\gamma$ is function of vector of tilt angles of all sectors i.e. $\boldsymbol{\theta}_{\text {tilt }}^{N}$ where $N=|\mathcal{N}|$, but for sake of simplicity of expression we will show this dependency only where necessary.

Given the small sector size we safely assume that a sector at most can have one RS within it at random location. We assume all RS have omnidirectional antennas and thus $0 d B$ gain. With this system model and assumptions, our problem can be stated as: optimize system wide antenna tilts to maximize the aggregate bandwidth normalised throughput i.e. spectral efficiency $\eta$ at access link of all the RS . Mathematically:

$$
\max _{\boldsymbol{\theta}_{\text {tilt }}^{N}} \eta\left(\boldsymbol{\theta}_{\text {tilt }}^{N}\right)=\max _{\boldsymbol{\theta}_{\text {tilt }}^{N}} \sum_{\forall s \in \mathcal{S}} \log _{2}\left(1+\gamma_{s}^{n}\left(\boldsymbol{\theta}_{\text {tilt }}^{N}\right)\right)
$$

where $\mathcal{S}$ is set of all points identifying locations of all RSs.

\section{Designing a SO Solution}

The formulation in (6) is a nonlinear large scale multi variable optimization problem. Its solution would require global cooperation among all BS's in the WCS and hence would not be distributed and therefore, would lack SO. In order to achieve a SO solution, this problem need to be first decomposed into subproblems, such that its solution can be executed locally in a distributed manner [5]. To this end, we propose to aim for a suboptimal solution as suggested in [5]. By not aiming for optimal solution, the tilt optimization can be done with local coordination only . To enable this localisation, we propose the concept of triplet. The triplet is fixed cluster of three adjacent and hence mutually most interfering sectors as shown in figure 2 . Let $\theta_{\text {tilt }}^{T_{i}}$ denote vector of tilt angle of sectors within $i^{\text {th }}$ triplet, now the local optimization problem to be solved and executed within a triplet is given as:

$$
\max _{\substack{\boldsymbol{\theta}_{\text {tilt }} \\ \boldsymbol{T}_{i}}} \hat{\eta}^{T_{i}}\left(\boldsymbol{\theta}_{\text {tilt }}^{T_{i}}\right)=\max _{\boldsymbol{\theta}_{\text {tilt }}^{T_{i}}} \sum_{\forall s \in \mathcal{S}_{i}} \log _{2}\left(1+\hat{\gamma}_{s}^{n}\left(\boldsymbol{\theta}_{\text {tilt }}^{T_{i}}\right)\right)
$$

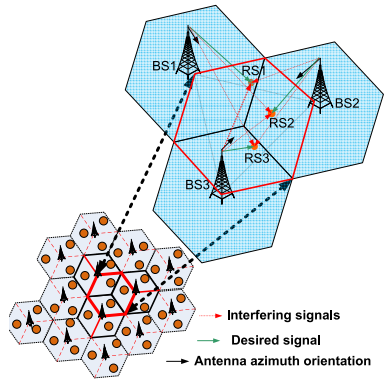

Fig. 2. A pictorial illustration of how system wide optimization of tilts can be decomposed into optimization of tilts within each triplet independently.

Whereas $\hat{\gamma}$ shows that SIR here is approximate SIR as it considers interference from the two most interfering adjacent sectors only. If $\hat{\eta}^{T}=C$ is throughput in a given triplet then (subscript $i$ dropped for generalisation over all triplets):

$$
C=\log _{2}\left(1+\hat{\gamma}_{1}^{1}\right)+\log _{2}\left(1+\hat{\gamma}_{2}^{2}\right)+\log _{2}\left(1+\hat{\gamma}_{3}^{3}\right)
$$

where postscript denote sector number and subscripts denote RS within a triplet, as shown in figure 2. Notice (7) is a small scale optimization problem now, as number of optimization parameters is only three and their range is also finite i.e. $0<$ $\theta<90$, so the solution of (7) can be easily determined using a non linear optimization techniques that can tackle a non convex optimization objective or even by exhaustive search. For sake of clarity, we drop the subscript tilt. Instead we use subscript to present the association with a sector in the triplet. Then the problem in (7) can be written in the standard form:

$$
\min _{\boldsymbol{\theta}}-C(\boldsymbol{\theta})
$$

subject to: $\quad g_{j}\left(\theta_{j}\right)<0, j=1,2,3$ where $\boldsymbol{\theta}=\left[\theta_{1}, \theta_{2}, \theta_{3}\right]$ and $g_{j}\left(\theta_{j}\right)=\theta_{j}-\frac{\pi}{2}$. Noticing that, the objective function is twice differentiable table and constraint is differentiable we can solve (9) using Sequential Quadratic Programming (SQP). To this end, the Lagrangian of constrained optimization problem in (9) can be written as:

$$
\mathcal{L}(\boldsymbol{\theta}, \boldsymbol{\lambda})=C(\boldsymbol{\theta})-\boldsymbol{\lambda}^{T} \boldsymbol{g}
$$

If $\hat{\boldsymbol{H}}$ denotes the approximate of the Hessian matrix $\boldsymbol{H}$, then we can define quadratic subproblem to be solved at $r^{t h}$ iteration of SQP as follows:

$$
\min _{\mathbf{w} \in R^{J}} \frac{1}{2} \mathbf{w}^{T} \hat{\mathbf{H}}(\mathcal{L}(\boldsymbol{\theta}, \boldsymbol{\lambda}))_{r} \mathbf{w}+\nabla C(\boldsymbol{\theta})_{r} \mathbf{w}
$$

subject to: $\quad w_{j}+\theta_{j_{r}}-\frac{\pi}{2}<0 \quad j=1,2,3$

At each iteration the value of $\hat{\mathbf{H}}$ can be updated using the Broyden-Fletcher -Goldfarb -Shanno (BFGS) approximation method. Once the Hessian is known the problem in (11) is a quadratic programming problem that can be solved using standard methods e.g. gradient projection method in [6].

Through the above steps of SQP, the problem in (7) can be solved within each triplet independently to determine the optimal tilt angles to be adapted and maintained by each triplet for given locations of RS within that triplet. The execution of 
these solutions in each triplet in the whole WCS independently, results in achievement of the system wide objective in (9), approximately. We call this framework SOT (SO of Tilts, as the basic idea of decomposing global objective into local objective is inspired from SO systems in nature [7]). Although the system wide optimal performance is neither aimed for nor achieved by SOT, however its just like the case that in nature SO systems do not aim for perfectly optimal objectives e.g. common cranes never fly in perfect $\mathrm{V}$-shape, but even maintaining a near $\mathrm{V}$-shape increases their group flight efficiency significantly [7]. Furthermore, as postulated in [5], one of the four main paradigms for designing SO into a system is that, for perfect SO perfect objectives should not be aimed for. So here the SO nature of the proposed solution is perfect but at cost of sub-optimal global objective.

\section{Numerical Results}

In order to demonstrate the gain SOT can yield, spectral efficiency for four different set of locations of RS's in a triplet are plotted, as shown in figure 3. A single triplet WCS with $\beta=4, B_{v}=10^{\circ}, B_{h}=70^{\circ}$, and cell radius of $600 \mathrm{~m}$, is assumed with BS and RS height of $30 \mathrm{~m}$ and $10 \mathrm{~m}$ respectively. Since, it is not possible to show a 4-D plot, therefore, one of the tilt angles $\theta_{\text {tilt }}^{3}$ is fixed to $0^{0}$ for ease of graphical illustration. Therefore, the actual gain achievable by the SOT by allowing it to set all the three tilts to optimal values, can be anticipated to be much higher then that shown in figure 3 .

Note that for each set of RS location the different tilt angles give optimal spectral efficiency. This highlights the practical need and use for SOT, particulary in scenarios of increasingly impromptu deployment of RS e.g. in emerging WCS like LTE-A. The gain of SOT is compared against two other sets of tilts i.e. arbitrarily low and high tilts of $\left(0^{0}, 1^{0}, 1^{0}\right)$ and $\left(0^{0}, 20^{0}, 20^{0}\right)$ respectively. For the first set of locations of RS (top left), the spectral efficiency with low tilting is $4.27 \mathrm{~b} / \mathrm{s} / \mathrm{Hz}$, and with high tilts it is even worse i.e. $4.24 \mathrm{~b} / \mathrm{s} / \mathrm{Hz}$. SOT on the other hand raises spectral efficiency to 5.36 i.e. a gain of over $1 \mathrm{bps} / \mathrm{Hz}$ is achieved by SOT in this case. Similarly, for second set of random RS locations, the gain of SOT is 0.7 and 0.6 compared to arbitrary low tilting and high tilting. Similar gains can be observed for third and fourth cases as well. It can be concluded from these results that, the SOT's exact amount of gain is dependent on locations of RS, and in general SOT can significantly enhance spectral efficiency on the access links for randomly located RS. Thus, SOT can substantially increase system-wide performance in emerging WCS like LTE and LTE-A that need SO functionalities, particulary to cope with impromptu deployment of RS. From practical implementation point of view, the main advantage of SOT is that it requires a negligible amount of only local signalling i.e. among the sectors within triplet to determine the location of RS. This signaling can be done through X2 interface in LTE or LTEA; and most importantly it can be done only when location of RS changes. Another advantage of SOT, that makes it more pragmatic, is that due to its highly localised nature it is very agile and can be implemented in autonomous and SO

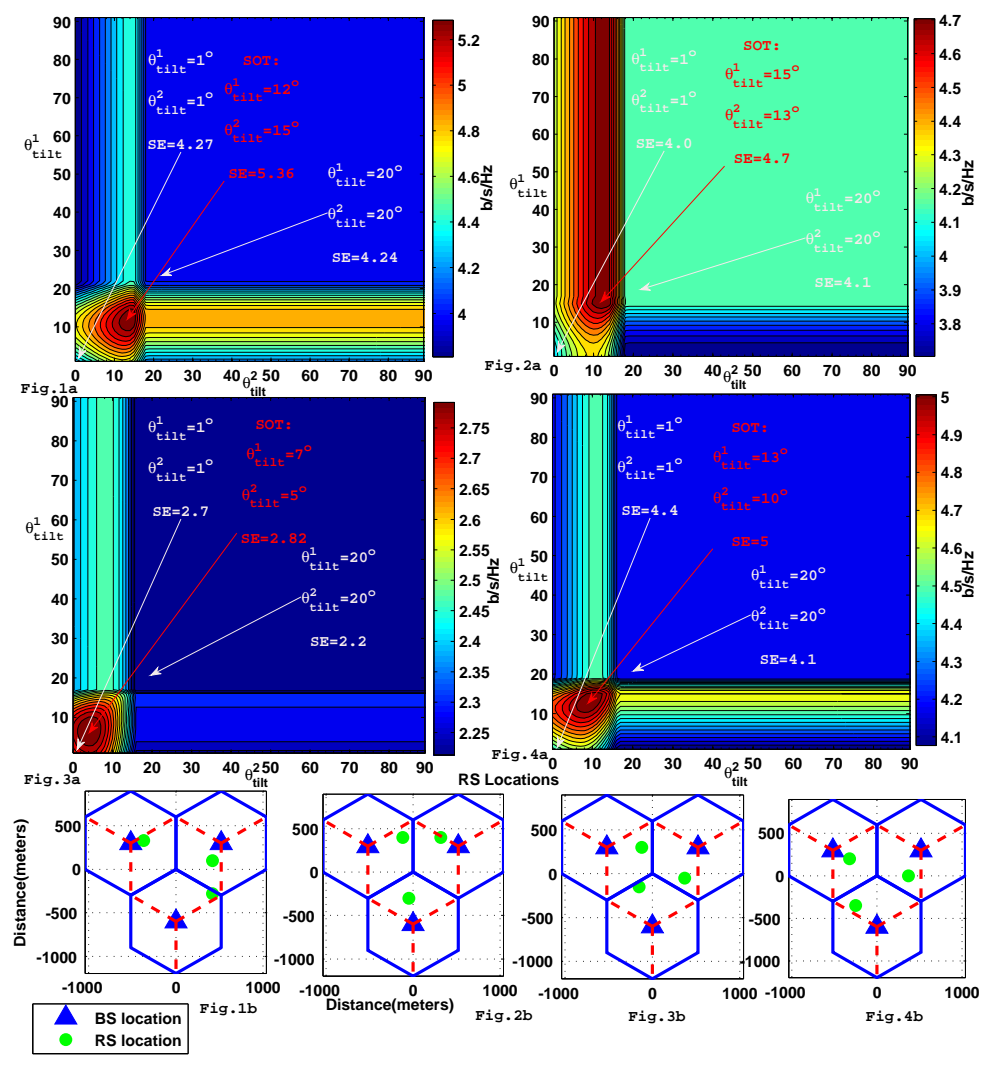

Fig. 3. Average spectral efficiency (SE) per link within a triplet i.e. $\frac{C}{3}$ is plotted as function of tilt angles of two sectors while $\theta_{t i l t}^{3}=0^{0}$.

fashion to cope with even mobile RS, as tilts can be changed electronically and remotely [2], [4].

\section{ACKNOWLEDGMENT}

This work has been performed in the framework of the ICT project ICT-4-248523 BeFEMTO, which is partly funded by the European Union. The authors would like to acknowledge the contributions of their colleagues from the BeFEMTO consortium.

\section{REFERENCES}

[1] A. Imran and R. Tafazolli, "Evaluation and comparison of capacities and costs of multihop cellular networks," 16th international conference on Telecommunications, pp. 160-165, 2009. [Online]. Available: http://portal.acm.org/citation.cfm?id=1700234.1700264

[2] V. Wille, M. Toril, and R. Barco, "Impact of antenna downtilting on network performance in GERAN systems," Communications Letters, IEEE, vol. 9, no. 7, pp. 598 - 600, july 2005.

[3] M. Amirijoo, L. Jorguseski, R. Litjens, and R. Nascimento, "Effectiveness of cell outage compensation in lte networks," in IEEE Consumer Communications and Networking Conference, Jan 2011, pp. 642 -647.

[4] I. Siomina, P. Varbrand, and D. Yuan, "Automated optimization of service coverage and base station antenna configuration in UTMS networks," Wireless Communications, IEEE, vol. 13, no. 6, pp. 16 -25, Dec. 2006.

[5] C. Prehofer and C. Bettstetter, "Self-organization in communication networks: principles and design paradigms," Communications Magazine, IEEE, vol. 43, no. 7, pp. $78-85$, july 2005.

[6] P. Gill, W. Murray, and M. H. Wright, Practical Optimization. London, Academic Press, 1981.

[7] P. B. S. Lissaman and C. A. Shollenberger, "Formation flight of birds," Science, vol. 168, no. 3934, pp. 1003-1005, 1970. [Online]. Available: http://www.sciencemag.org/cgi/content/abstract/168/3934/1003 